\title{
Review of laser diagnostics at the Vulcan laser facility
}

\author{
Ian Musgrave, Marco Galimberti, Alexis Boyle, Cristina Hernandez-Gomez, Andrew Kidd, Bryn Parry, \\ Dave Pepler, Trevor Winstone, and John Collier \\ Central Laser Facility, Science and Technology Facilities Council, RAL, Didcot, Oxfordshire OX 11 OQX, UK \\ (Received 1 May 2015; revised 6 July 2015; accepted 31 July 2015)
}

\begin{abstract}
In this paper we review the provision of the laser diagnostics that are installed on the Vulcan laser facility. We will present strategies for dealing with the energy of high energy systems and with ways of handling the beam sizes of the lasers. We present data captured during typical experimental campaigns to demonstrate their reliability and variation in shot to shot values.
\end{abstract}

Keywords: calorimeter; high power laser; laser diagnostics; pulse energy

\section{Introduction}

The Vulcan laser facility of the Central Laser Facility is located in the UK. It is an open access laser facility operated ostensibly for the UK academic community for the purposes of studying laser-matter interactions ${ }^{[1-3]}$. A 3D artist's impression of the Vulcan laser facility is shown in Figure 1. All the seed oscillators for the Vulcan laser facility are housed in the front end. They are then injected into the laser amplifier bay before being sent to the relevant target area. Vulcan has two operational target areas: Target Area Petawatt (TAP) has a $1 \mathrm{PW}$ (500 J and $500 \mathrm{fs}$ ) peak power beam combined with a single long pulse beam (250 J in up to $6 \mathrm{~ns}$ ) and Target Area West (TAW) is a flexible target area with up to eight beams which can be operated in either all long pulse or a combination of six long pulse beams and two short pulse beams, one at $100 \mathrm{~J}$ in 1 ps and the other operating in either $100 \mathrm{~J}$ at $1 \mathrm{ps}$ or $300 \mathrm{~J}$ at $10 \mathrm{ps}$. Vulcan uses flash-lamp-pumped $\mathrm{Nd}$ :glass for power amplification, and thermal effects within the amplifiers limit the shot repetition rate to two full energy shots per hour.

A schematic of the short pulse beam line for TAP is shown in Figure 2; for a detailed description of the beam line see Musgrave et al. ${ }^{[4]}$. Here, we summarize the pertinent points for our discussion. The seed pulse is generated by a commercial Ti:sapphire oscillator, the output of which is directed to a single stage of ps optical parametric chirped pulse amplification (OPCPA) where it is amplified to $100 \mu \mathrm{J}$. The pulses are then stretched to $4.5 \mathrm{~ns}$ in a double decker

Correspondence to: I. Musgrave, Central Laser Facility, Science and Technology Facilities Council, RAL, Didcot, Oxfordshire OX 11 0QX, UK. Email: ian.musrgave@stfc.ac.uk
Öffner stretcher. Following this are three further stages of OPCPA to achieve $>60 \mathrm{~mJ}$ of energy. These pulses are then amplified in a mixed silicate and phosphate glass rod amplifier chain before final amplification in a phosphate disc chain. The pulses are finally compressed before being directed to the target using an off-axis parabola.

A similar beam line which shares many components for TAW $^{[5]}$ is shown in Figure 3. Again, a seed oscillator is stretched to several nanoseconds before being amplified in a glass laser chain that amplifies the pulses up to $500 \mathrm{~J}$ depending on the requirements of the experimental campaign. After amplification the beams can be compressed in two separate compressors. The long pulse beam lines are amplified in a similar fashion to the beams for TAW, using an all-phosphate glass rod amplifier chain followed by a phosphate disc amplifier chain.

With this degree of flexibility the diagnostic challenges that are presented in characterizing the laser pulse are diverse. Fundamental to being able to interpret and model the results obtained from the laser-matter interactions is the knowledge of the laser pulse parameters. In addition, diagnostics are required to monitor the different components of the laser to improve performance and provide better control. Care is needed when designing and implementing diagnostics packages so that they do not unduly alter the parameters of the pulse being measured. A significant challenge when measuring the pulses of high power lasers is how to image very large aperture beams often in constrained spaces without degrading the beam quality. In addition, this process cannot be allowed to introduce too much B-integral which might compromise the temporal shape measurement of a short pulse. Our post-compression laser diagnostics are designed to introduce a B-integral of less than one. 


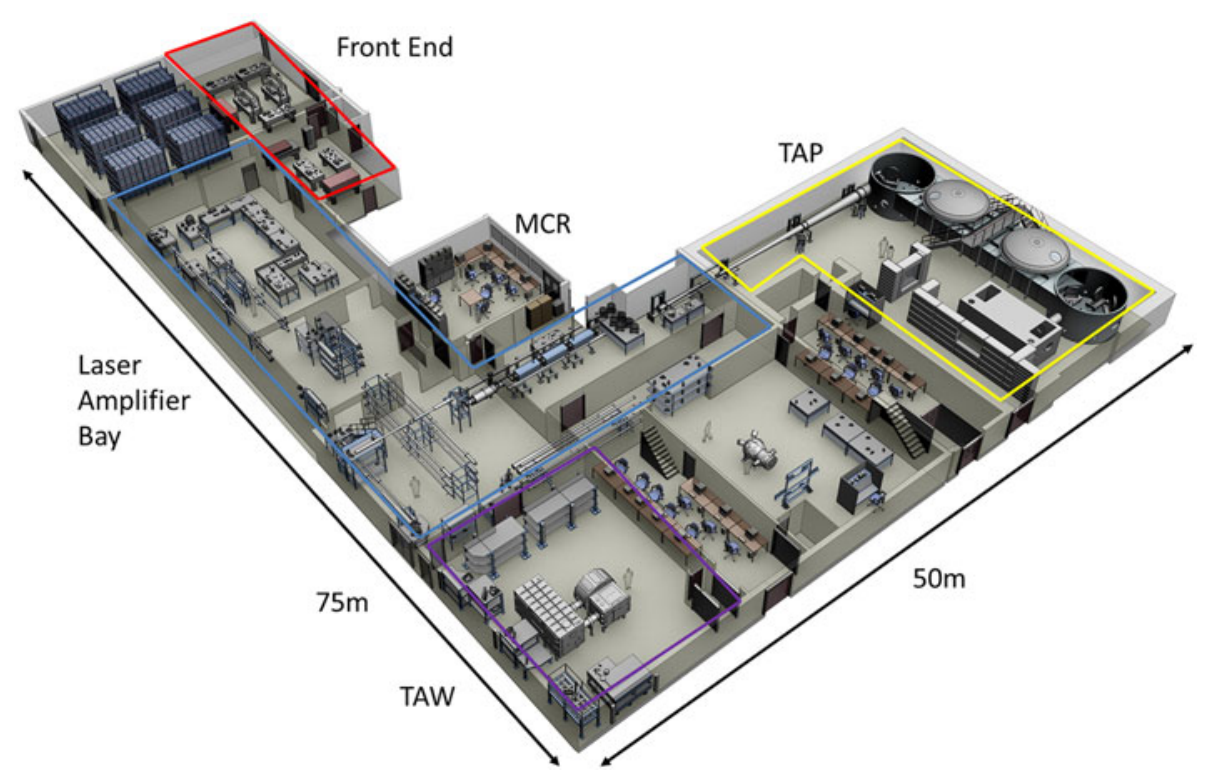

Figure 1. Artist's 3D impression of the Vulcan laser facility.

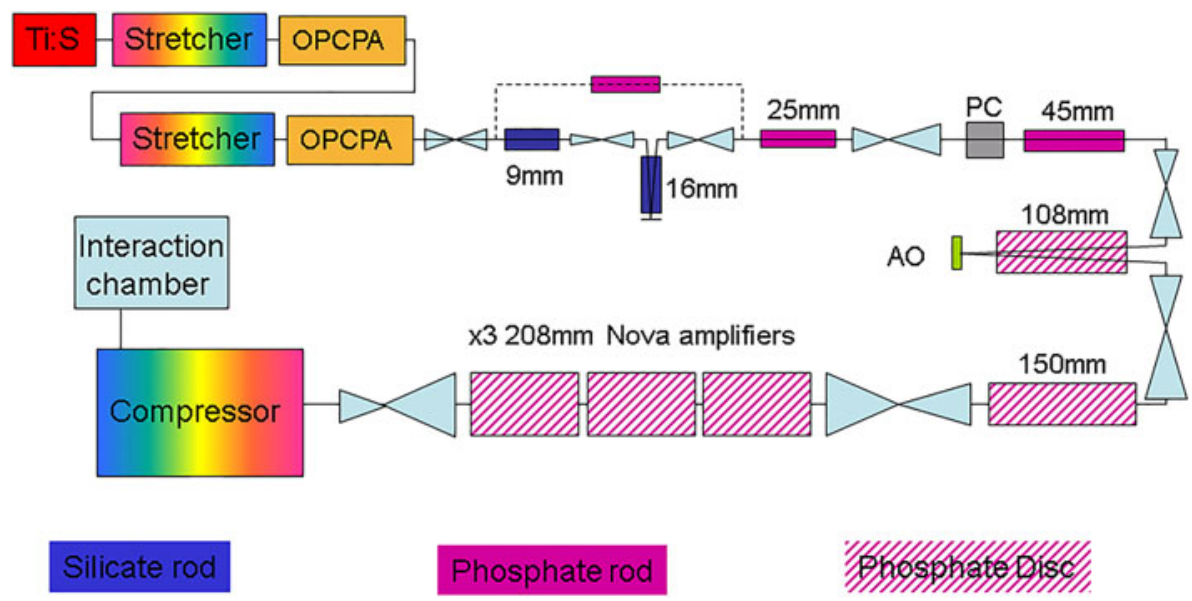

Figure 2. Schematic of the Vulcan beam line for TAP.

In this paper we will present the strategies that we have developed to enable us to monitor the performance of the laser. These include technologies that we employ to attenuate the energy of the laser pulse in the diagnostics packages so that damage does not occur. They also demonstrate how we prevent the diagnostics from becoming influenced by Bintegral effects.

\section{Front end diagnostics}

Depending on the laser parameters required for a particular experimental campaign there are a variety of oscillators that might be used. These are installed in the Vulcan front end, with each oscillator having a dedicated diagnostics package to monitor its performance. Each short pulse oscillator has a spectrometer and the long pulse sources have pulse shape diagnostics. The pulse shape is measured using a fibre-coupled photo-diode onto an oscilloscope whose traces can be captured on a local PC. An example of the output diagnostics package that is used to monitor the oscillator used to seed the laser for TAW is shown in Figure 4. This figure shows the near- and far-fields at the input and exit of the stretcher and the spectrum. The front end also houses the pre-amplifiers and stretcher for the petawatt beam; here we monitor the near- and far-fields for the different stages and the output energy and spectrum. To monitor the performance of these systems the cameras are operated in triggered mode and capture an image on the full laser shot. For all of these diagnostics packages it is sufficient to employ the leakage through a mirror to attenuate the beam. However, for the OPCPA diagnostics care is required with the filters to ensure 


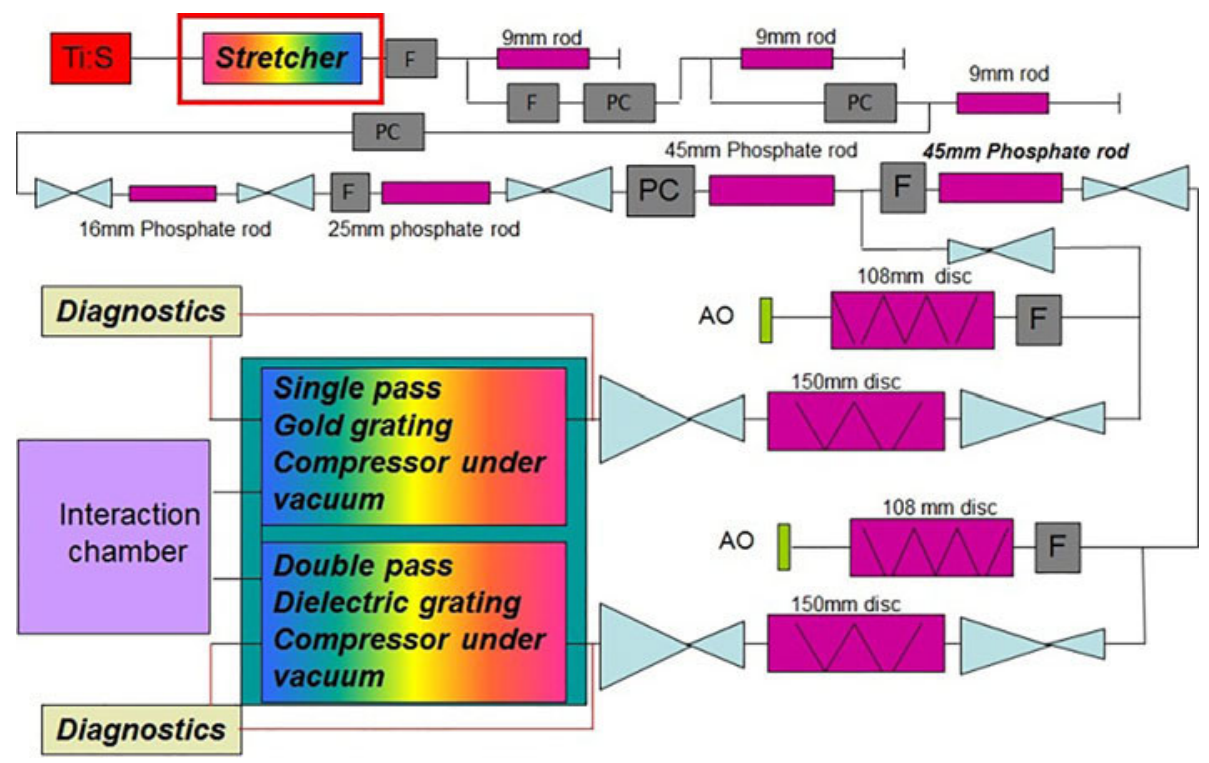

Figure 3. Schematic of the Vulcan short pulse beam lines for TAW.

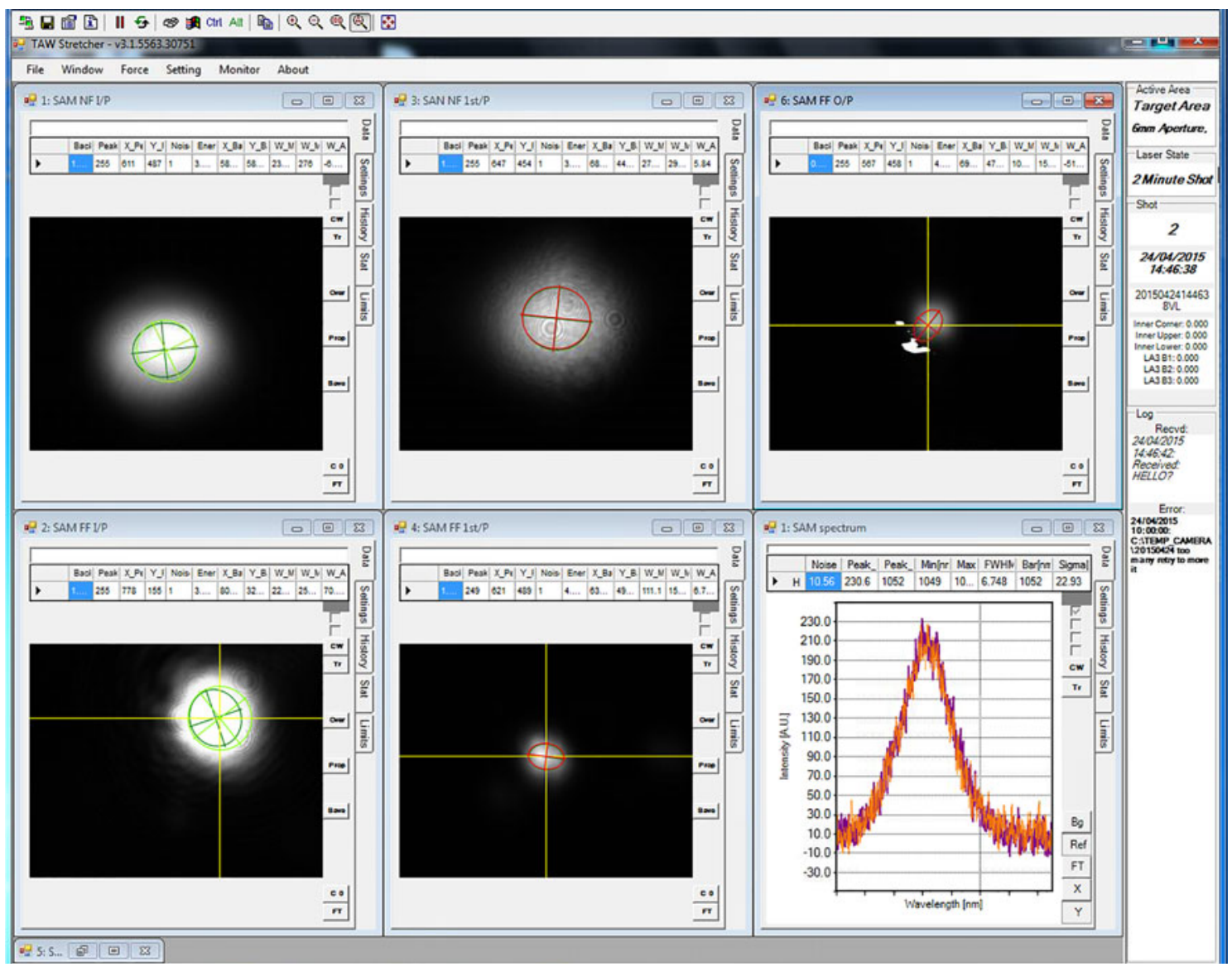

Figure 4. Example of the output of a diagnostics package from the TAW oscillator.

that there is sufficient attenuation of the pump wavelength which is $532 \mathrm{~nm}$ without unduly attenuating the signal at $1053 \mathrm{~nm}$. The outputs from all of these diagnostics packages are sent to the laser main control room so that they can be monitored by the laser operation team. A summary table of the different diagnostics is shown in Table 1. 


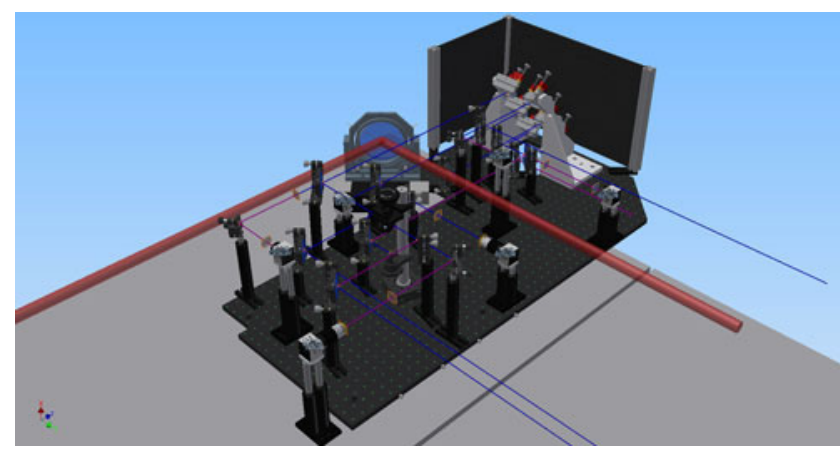

Figure 5. Diagnostics package to monitor the injection into the laser bay.

Table 1. Summary of front end diagnostics.

\begin{tabular}{lll}
\hline Component & Diagnostics & Regime 3 \\
\hline SP oscillator & $\mathrm{NF}, \mathrm{FF}$ and Spec & Continuous \\
LP oscillator & $\mathrm{NF}, \mathrm{FF}, \mathrm{TS}$ and E & $10 \mathrm{~Hz}$ \\
Stretcher input & $\mathrm{NF}$ and FF & Continuous \\
Stretcher output & $\mathrm{NF}$ and FF & Continuous \\
OPCPA pre-amp & $\mathrm{NF}^{\mathrm{a}}, \mathrm{FF}^{\mathrm{a}}, \mathrm{E}$ and Spec & $\mathrm{b}$ \\
\hline
\end{tabular}

Notes: SP, short pulse; LP, long pulse; NF, near-field; FF, far-field; TS, temporal shape; Spec, spectrum; E, energy.

${ }^{a}$ For each gain stage and at output.

${ }^{\mathrm{b}}$ At output.

\section{Laser amplifier bay diagnostics}

There are a number of diagnostic packages that are used to characterize the beam lines and the pulses as they are amplified. These diagnostics have a variety of purposes and occur at staged intervals through the laser system. The first of these is used to monitor the injection of the different seed sources from the front end into the laser amplifier bay. There is a single channel per oscillator comprising a near- and far-field (Figure 5). These diagnostics only experience the outputs from the oscillators, and so the challenge for these is to ensure that they have sufficient light to activate the CCD cameras and that for the pulsed systems they only image a single pulse, rather than show the accumulation of a number of pulses.

Figure 6 shows an example of a diagnostics package that is used to monitor the injection of the laser pulses into one of the rod amplifier chains of Vulcan. The beam for the diagnostics channel is taken through the leakage of a polarizing beam splitter. It is at this point that the different oscillators are combined. The beam that has gone through the splitter to the diagnostics package is then further split to provide channels for near- and far-field image capturing, an input into an optical fibre for a streak camera to monitor the pulse shape, a spectrometer to measure the spectrum and an additional channel for a partially compressed synchronized optical trigger. A continuous wave (CW) laser beam used to check the alignment of the laser chain is also injected at this point. This diagnostics package is designed to handle the inputs from up to four different seed sources that could be injected at this point and is crucial for checking their overlap. The typical pulse energy at this point is $2-5 \mathrm{~mJ}$ with pulse durations of $0.5-6 \mathrm{~ns}$ and a beam diameter of $4 \mathrm{~mm}$.

After the rod amplifier chain there is another set of diagnostic packages which are shown in Figure 7. At this position the near- and far-fields are captured, as are the spectra of the two beam lines and the energy. At this point in the laser chain, the diagnostics packages have to be able to operate for $\mathrm{CW}$ alignment beams up to the multiple joule level which can be generated from the rod amplifier chains. The beam diameter at this point is $50 \mathrm{~mm}$. The beams are injected into the diagnostics package through the leakage of a $97 \%$ reflective mirror. Most of the energy is directed to a calorimeter for energy measurement. A motorized filter wheel containing a range of neutral density filters is used to attenuate the beam as needed to keep the energy incident onto the cameras and spectrometer nearly constant.

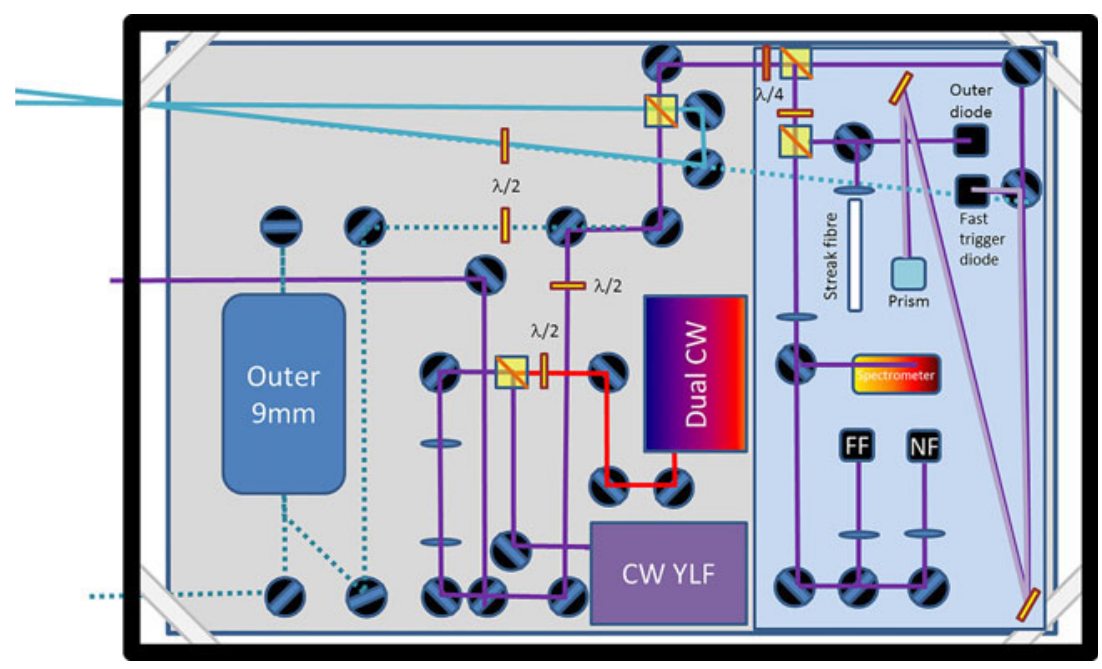

Figure 6. Diagnostics package for the injection into the rod chain. 


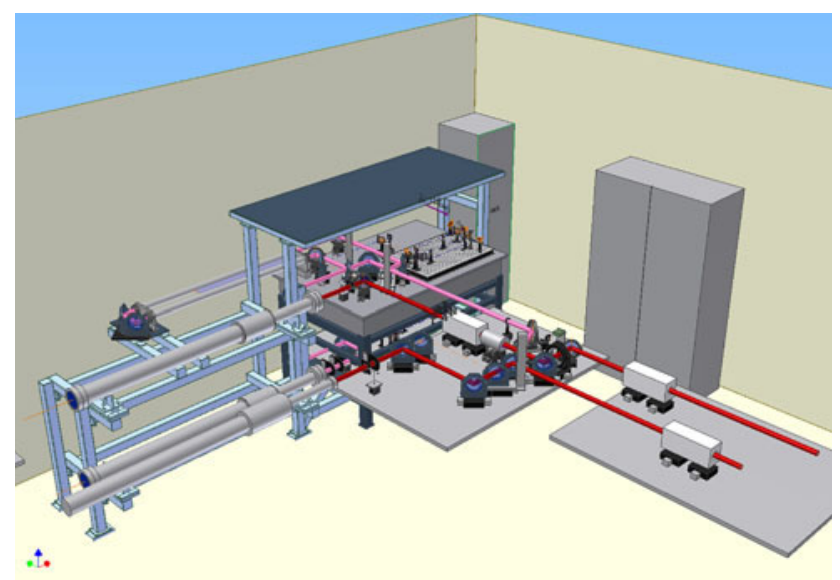

Figure 7. Diagnostics package at the end of the rod amplifier chain.

The motorized filter wheel is controlled by the diagnostics software package which is running locally to the diagnostics suite, but which is in communication with the overall control software for the laser facility.

After the rod amplifier chains, the beams are further amplified in disc amplifiers. There are a range of diagnostics packages that follow the same design principle for apertures of 108,150 and $200 \mathrm{~mm}$ beam diameters. The near-field cameras are positioned so that they image the final disc amplifier. Here, the challenges come from downsizing the beam to match the CCD camera chip size of typically $4 \times 5 \mathrm{~mm}$ and safely attenuating the energy. In addition to the usual near- and far-fields, spectra and energy, some of these diagnostics packages also incorporate wavefront sensors to provide feedback to the adaptive optics (AO) systems in the laser chain. A typical layout of such a diagnostics package is shown in Figure 8. The AO is optimized before a full energy disc shot by propagating the alignment $\mathrm{CW}$ beam through the laser chain and using in-house-developed software to improve the wavefront and correct for any thermal aberrations in the beam. Once the $\mathrm{AO}$ has been optimized it is held in that position until the next time the process is repeated.

Energy handling is achieved by using a combination of glass plates and mirrors that can be switched without changing the alignment or focusing of the system. An example of this arrangement is shown in Figure 8; the incident beam is brought to focus and if the low energy or $\mathrm{CW}$ alignment mode is required then mirrors are used to direct the beam to the near- and far-field cameras. For a full energy shot the mirrors are replaced with glass wedges that are used to attenuate the beam as it propagates to the cameras. Using this scheme the energy can be attenuated by nearly three orders of magnitude without introducing a deleterious Bintegral. Further attenuation could be achieved if antireflective coated optics were used instead of the glass plates. Care needs to be exercised when aligning this arrangement of mirrors to equalize the relative path lengths to ensure that the subsequent focal positions are in the same plane for both the high and low power modes.

The downsizing of the beams can be achieved using a positive-negative combination of lenses to prevent the requirement of focusing in the air or under vacuum; this is shown in Figure 9. In this scheme the diagnostics beam is incident from the left and is already being focused. The negative lens collimates this beam at approximately $50 \mathrm{~mm}$ in diameter. A beamsplitter is then used to direct most of the light to the energy calorimeter and a fibre for pulse shape measurement. A nitrogen-driven pneumatic shutter with a fixed neutral density filter is used to attenuate the beam for on the shot and $\mathrm{CW}$ operation. For $\mathrm{CW}$ and low power operation the shutter is out for high power operation the shutter is in with suitable filtration to prevent damage to the cameras. In this way a single diagnostics package can have a dynamic range of at least four orders of magnitude. After the shutter the beam is imaged onto a near-field camera and a far-field camera. The far-field systems typically have a fieldof-view of $250 \mu \mathrm{rad}$.

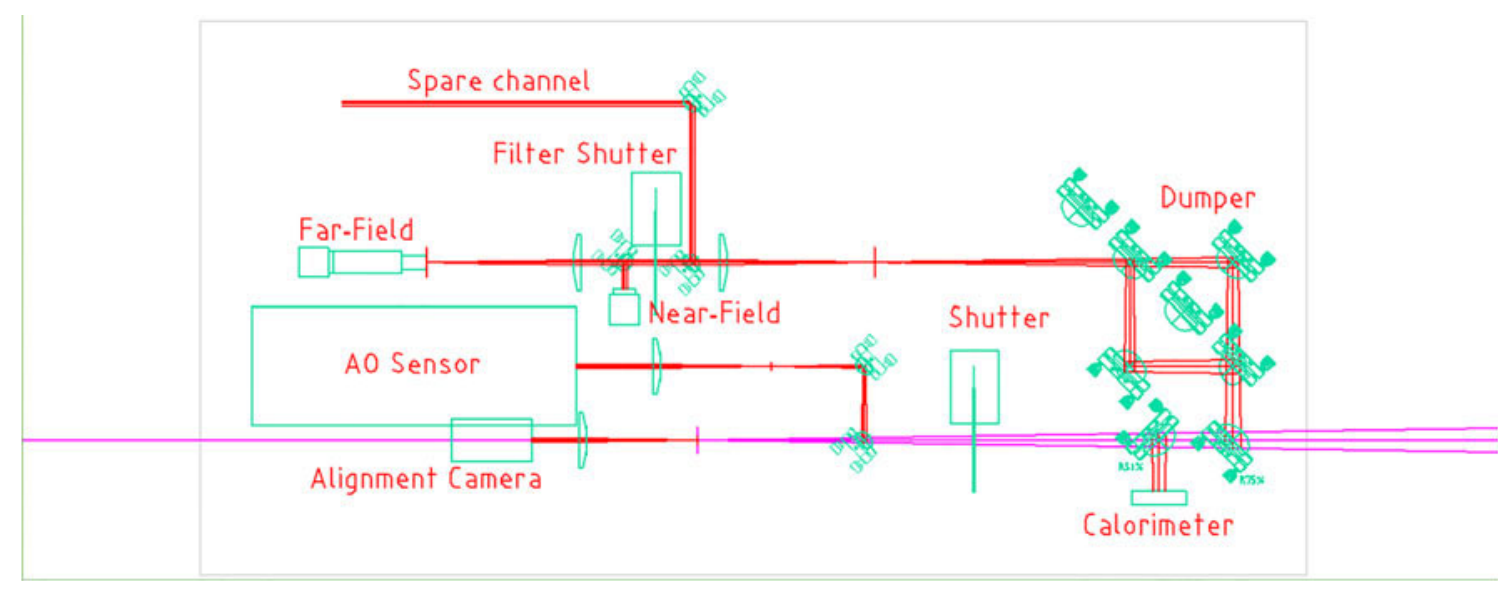

Figure 8. Schematic of the diagnostics package used at the end of the disc amplifier chain. 


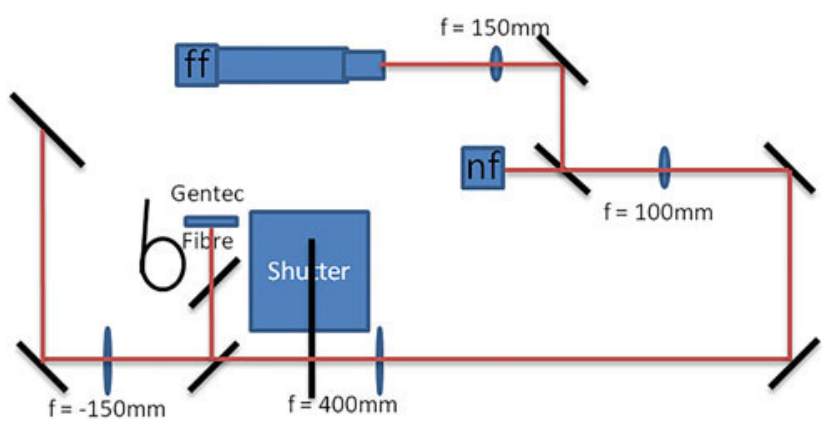

Figure 9. Schematic of the diagnostics package at the output of the $108 \mathrm{~mm}$ beamlines. The beam is incident from the left and is being focused by a lens (not shown).

Table 2. Summary of the laser bay diagnostics.

\begin{tabular}{lll}
\hline Component & Diagnostics & Regime \\
\hline Injection & NF, FF & Co, Trg, PS, OS \\
Rod chain input & NF, FF & Co, Trg, PS, OS \\
Rod chain output & NF, FF, SP and E & Co, Trg, PS, OS \\
108 mm disc output & NF, FF, TS and E & Co, Trg, PS, OS \\
$150 \mathrm{~mm}$ disc output & NF, FF, SP and E & Co, Trg, PS, OS \\
$200 \mathrm{~mm}$ disc output & NF, FF, SP and E & Co, Trg, PS, OS \\
\hline
\end{tabular}

Notes: NF, near-field; FF, far-field; TS, temporal shape; SP, spectrum; E, energy; Co, continuous; Trg, triggered; PS, pre-shot; OS, on shot.

Table 2 summarizes the different diagnostics that are installed throughout the laser amplifier bay. We also note those diagnostics that are used prior to the laser shot to confirm alignment and those that are used on the shot for laser pulse characterization and archiving.

\section{Target Area Petawatt diagnostics}

When originally commissioned, the laser diagnostics installed in TAP comprised a single full aperture diagnostics channel described in further detail below. The system operated with single shot uniaxial autocorrelators ${ }^{[6]}$ that were used in both the dispersion and non-dispersion planes to measure the pulse length. These diagnostics had insufficient temporal window for some experimental campaigns and were very sensitive to far-field misalignment; therefore, a single shot near-field autocorrelator was developed with a larger temporal window to diagnose the pulses. To further improve the reliability of the diagnostics an additional subaperture beam was installed with a low B-integral line for pulse length measurement.

The compressor chamber for TAP and the diagnostics beam paths are shown in Figure 10. The incident beam (red path) is expanding as it enters the compressor chamber from the final vacuum spatial filter and is collimated by lens L1. After L1 the beam is incident on mirror M1 which is coated to be highly reflecting (HR) at $45^{\circ}$ at $1053 \mathrm{~nm}$ and directs the beam to the first grating, G1. Then, G1 diffracts the beam to grating G2. After G2 the beam is directed to mirror M2

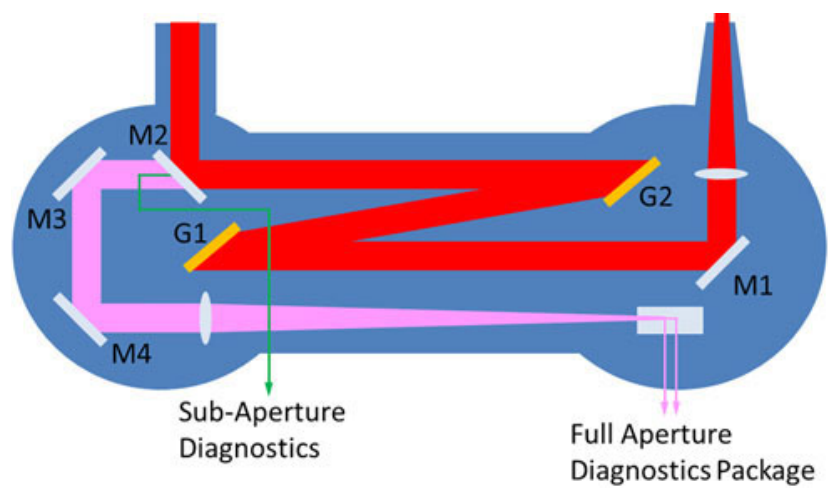

Figure 10. Schematic of the Vulcan short pulse diagnostics in TAP. The main pulse follows the red path, the full aperture beam the pink path and the sub-aperture diagnostics the green path.

Table 3. Summary of target area laser diagnostics.

\begin{tabular}{lll}
\hline Component & Diagnostics & Regime \\
\hline Full aperture & NF, FF, Con, SAC & Co, Trg, PS, OS \\
Sub-aperture & NF, FF, SAC, LAC, and SP & Trg, OS \\
TAW LE & NF, FF, LAC and SP & Co, Trg, PS, OS \\
TAW HE & NF, FF, LAC and SP & Co, Trg, PS, OS \\
\hline
\end{tabular}

Notes: NF, near-field; FF, far-field; SP, spectrum; SAC, small window autocorrelator (10 ps); LAC, large window autocorrelator (60 ps); Con, contrast diagnostic; Co, continuous; Trg, triggered; PS, pre-shot; OS, on shot; LE, low energy; HE, high energy.

which directs most of the beam into the interaction chamber. A summary of the diagnostics used in these two diagnostics packages is shown in Table 3 .

\subsection{Full aperture diagnostics}

A schematic of the diagnostics beam is shown in Figure 10 (pink path). The full size beam diagnostics take the leakage through the back of the mirror (M2) at the output of the compressor. The M2 mirror transmits approximately $2 \%$ of the incident energy (of the order of $10 \mathrm{~J}$ ) in a nominally 500 fs pulse through a $100 \mathrm{~mm}$ thick BK-7 glass. The beam is then incident on two $45^{\circ}$ mirrors that are coated to be $\mathrm{HR}$ at $22.5^{\circ}$, thereby attenuating some of the energy. The beam then passes through a focusing lens where the beam is focused under vacuum. After focusing the beam is attenuated using the scheme shown in Figure 8 . The beam that is reflected off the glass plate is directed to near- and far-field cameras and an autocorrelator. The higher energy beam is directed to a photo-diode and water cell combination for contrast measurement. This arrangement has a dynamic range of approximately 10 orders of magnitude; a typical trace is shown in Figure 11. This figure shows the output of the photo-diode plotted on both linear and logarithmic scales. As can be seen, it does not have sufficient dynamic range to measure the amplified spontaneous emission on the shot. The temporal resolution of this setup is the oscilloscope used to capture the trace from the photo-diode and this limits 


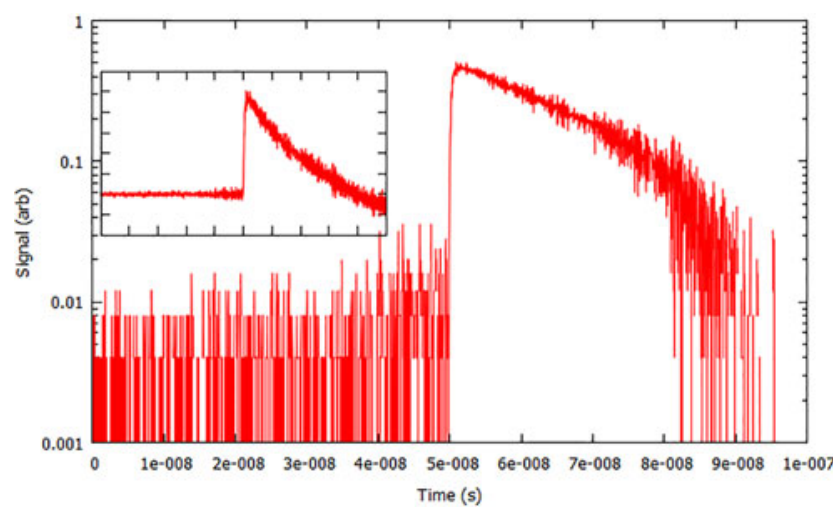

Figure 11. Oscilloscope trace of contrast diode capture on a shot.

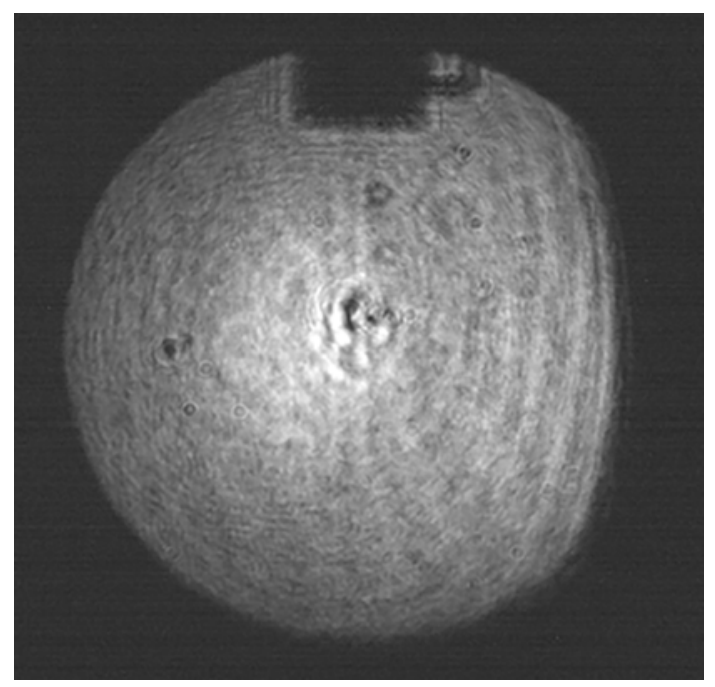

Figure 12. Laser near-field recorded on the full aperture diagnostics.

the validity of the data to approximately 200 ps before the arrival of the pulse. Measurement of the close in contrast requires the use of a scanning third-order cross-correlator. Our reported measurements of the close in contrast ${ }^{[4]}$ have been achieved using a by-pass line and relaying the beam from the interaction chamber.

Spatially, the full size diagnostics are very powerful tools for near-field characterization, due to the use of image relaying from the second grating. This also enables the diagnosis of damage to the optics in the compressor. An example of the near-field recorded on a full energy laser shot is shown in Figure 12. The beam size was originally $0.6 \mathrm{~m}$ in diameter, the shadow at the top of the beam is a beam block and motor drive cable placed after M2 for the sub-aperture diagnostics line, and the feature in the centre of the image is caused during the writing of the grating during manufacture. The beam block is permanently in position to prevent the sub-aperture beam from propagating through the full aperture diagnostics channel which might lead to damage. The clip on the right-hand side of the image is due

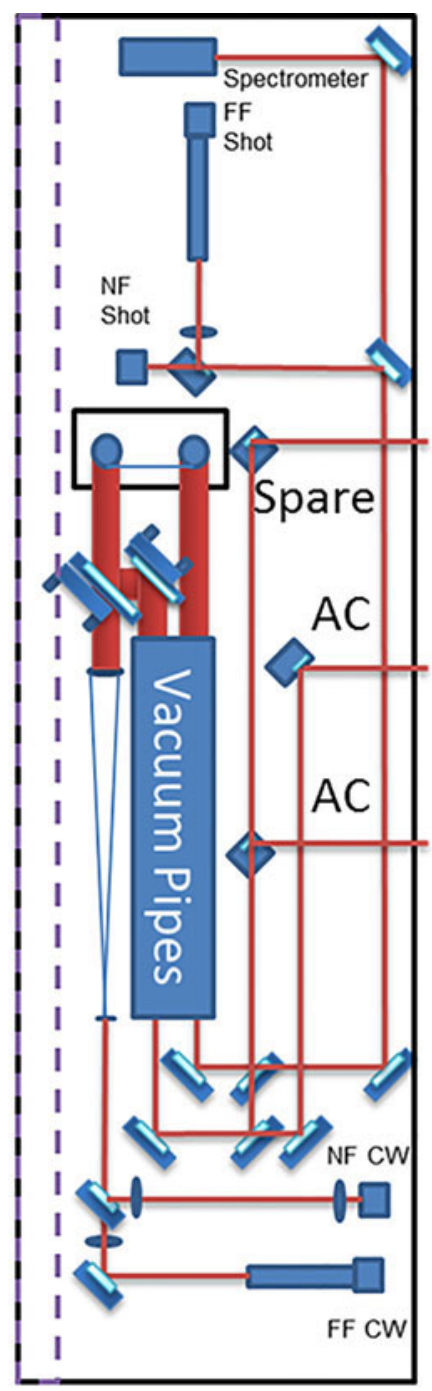

Figure 13. Layout of the low sub-aperture diagnostics.

to the transmission through M2; it is caused by the diagnostic beam being incident on the bezel holding M2. However, the sampling method leads to undesirable nonlinear effects, as the beam passes through M2 and the diagnostics lens. The B-integral gives a good indication of whether a pulse will be affected by nonlinear effects, and for this diagnostics line it is calculated to be as high as $4 \mathrm{rad}$. In the past, selfphase modulation (SPM) has been observed while trying to measure the spectrum on the full size diagnostics channel.

\subsection{Sub-aperture diagnostics channel}

To mitigate the $\mathrm{B}$-integral issue a hole was drilled through the M2 mirror during a reworking of the optic prior to coating, and a route was designed and installed to take a sub-aperture M2 beam outside of the compressor chamber to a suite of diagnostics. This enables diagnosis of the short pulse without B-integral effects. The approximately $50 \mathrm{~mm}$ 


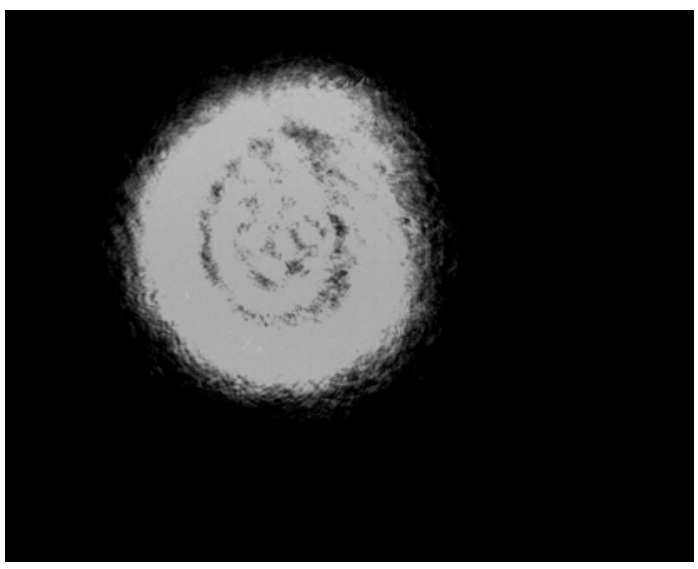

Figure 14. Near-field incident onto the sub-aperture diagnostics.

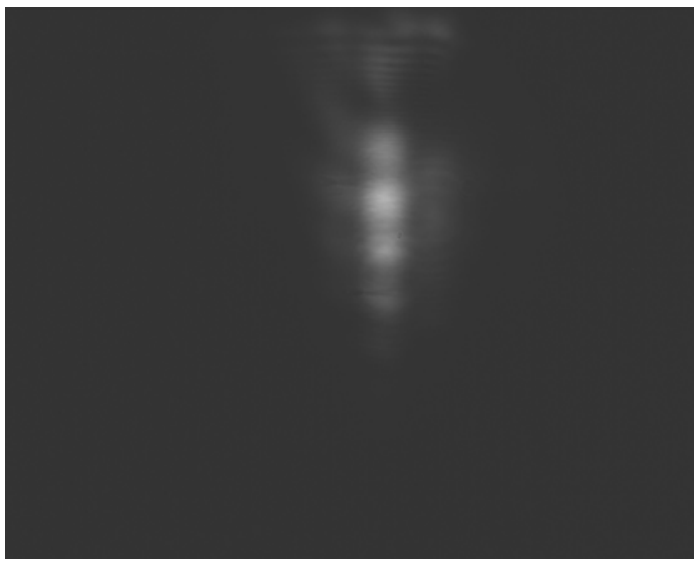

Figure 15. Image captured by the near-field autocorrelator in the subaperture diagnostics package.

diameter beam is incident onto a series of mirrors that direct the beam out of the compressor vacuum chamber, as shown in Figure 10 (green path). A glass plate is used to attenuate the beams before they leave the vacuum window; they are then relayed onto the autocorrelators. Due to space constraints, this diagnostics package is mounted vertically, as shown in Figure 13. There are three possible diagnostics channels; the first is used for a single shot near-field autocorrelator and the second is for a large window autocorrelator that has a $60 \mathrm{ps}^{\text {window }}{ }^{[7]}$. To ensure that the $\mathrm{B}$-integral is kept to a minimum there is no image relaying of the beam as it propagates within the compressor chamber; consequently, the near-field of the beam develops diffraction rings over the propagation length. An example of the near-field is shown in Figure 14. The near-field autocorrelator effectively averages across the beam, and therefore these rings are not present in the captured autocorrelation images, an example of which is shown in Figure 15. The autocorrelation trace is then calculated by taking a lineout across the image, and an example of one of the autocorrelation traces recorded on a full energy disc shot is shown in Figure 16. In addition to

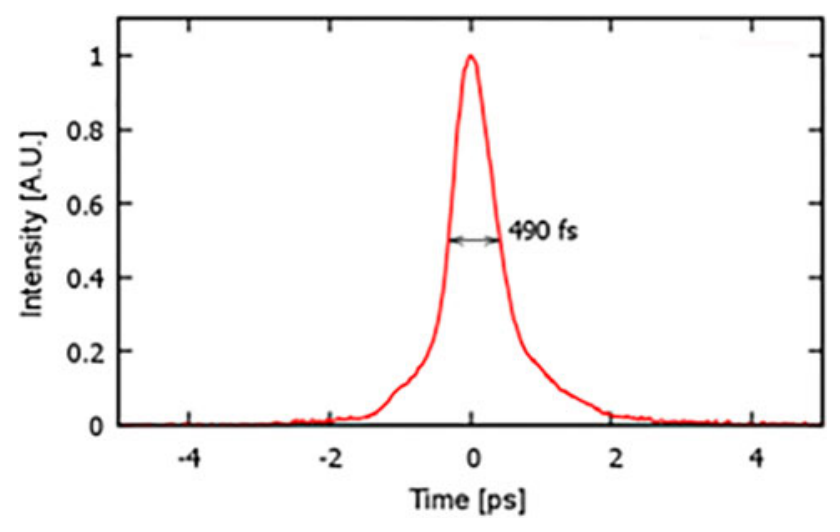

Figure 16. Example of an autocorrelation trace recorded using the subaperture diagnostics line.

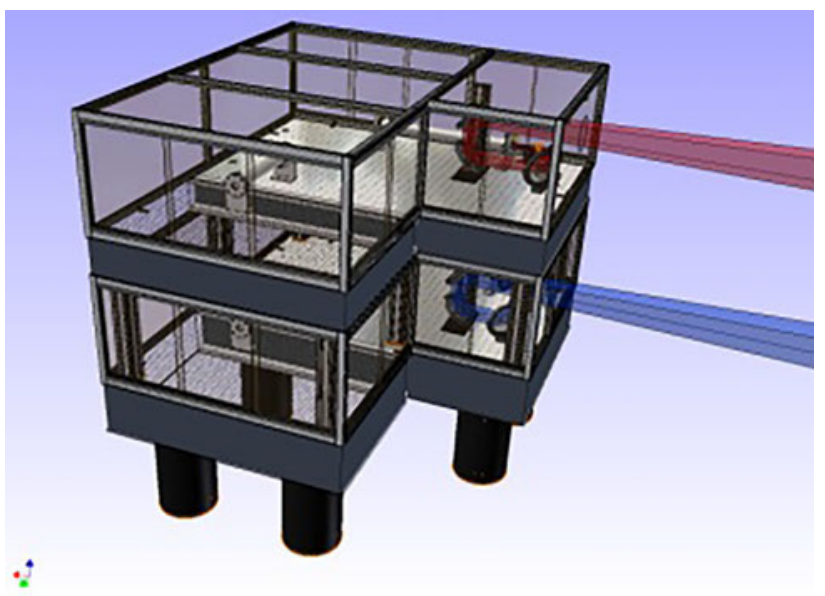

Figure 17. Image of the post-compression diagnostics suite in TAW.

the much reduced B-integral effect, the pointing stability into this diagnostics package is better, so that the performance of the autocorrelators is improved. The position of the hole is such that it is at the bottom of the beam; this area of the beam is in the shadow of optics that are positioned subsequent to this which are used to pick off a portion of the beam for an optical probe. Consequently, any diffraction rings that might be present in the main beam are then blocked before they are incident on the final focusing optic.

\section{Target Area West diagnostics}

The compressor arrangement in TAW is different from that in TAP because it is built on two levels in order to make best use of space. As a consequence, the post-compression diagnostics are on two levels as well, as shown in Figure 17. The diagnostic beams for the two compressors are extracted in the same way by using the transmitted leakage through the final turning mirror between the compressor and the interaction chamber. After the mirrors, two lenses 


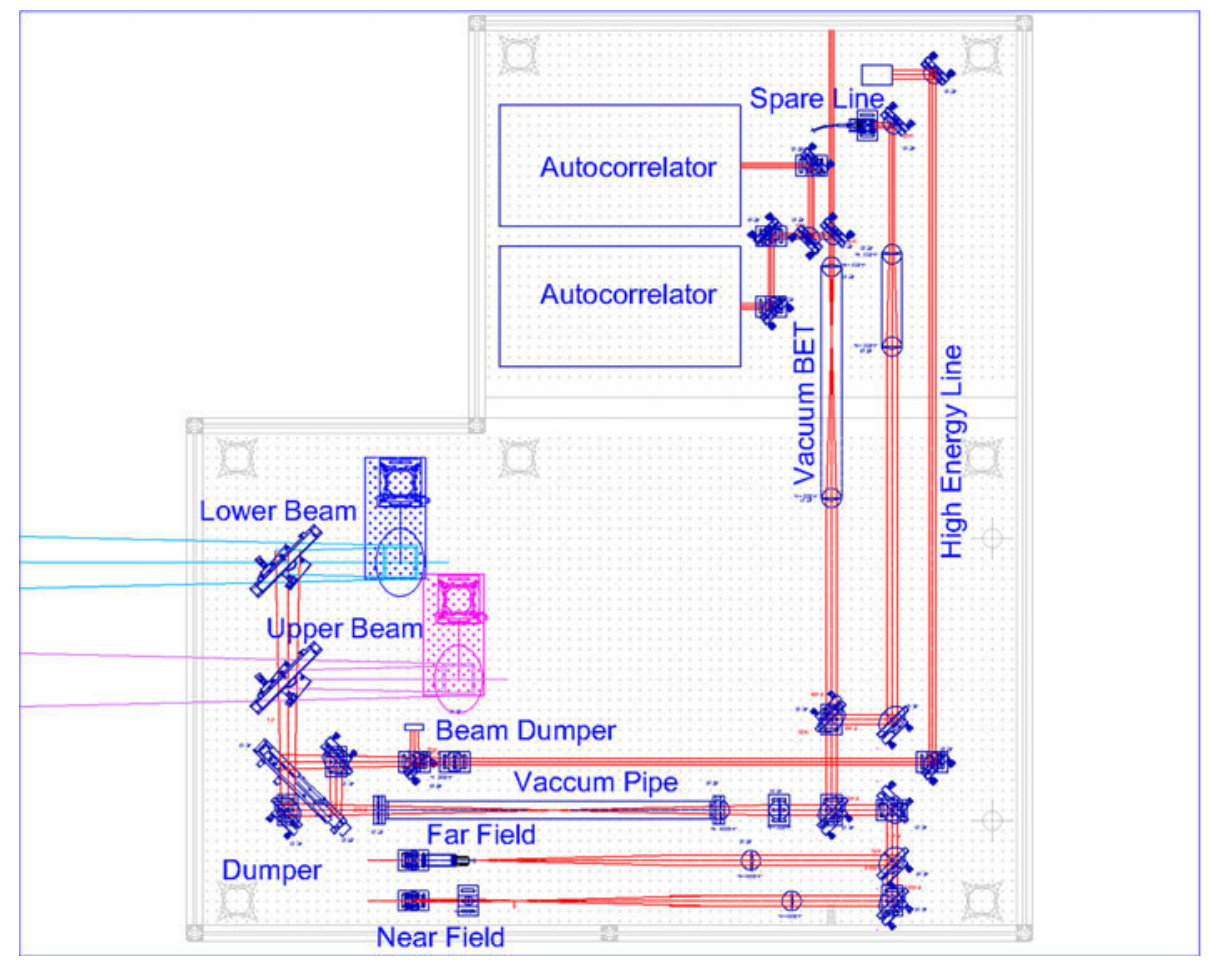

Figure 18. Schematic of the layout for the short pulse diagnostics in TAW.

(one for each beam) are used to focus the beam down as part of a de-magnifying telescope. These two large lenses are inside the compressor vacuum chamber, and the collimating lenses are located on the diagnostics table at air. The reflectivity of the two mirrors is $99 \%$ on the high energy beam and $98 \%$ on the low energy beam, in order to keep the B-integral less than 1. Since the two diagnostic beams have similar characteristics, we have designed the diagnostics to have the same layout, except for the first three mirrors. The layout used is shown in Figure 18. Both tables are positioned so that they are slightly lower than the beam heights. Looking at just the lower beam, the incoming beam from the left is reflected down and then backward by two mirrors. Another mirror directs the beam to the energy dumper. The layout of the dumper is exactly the same as the one used in Figure 8. However, we now collect part of the dumped beam which is re-collimated by a negative lens and sent to a high energy line which is useful for diagnostics like a photo-diode nanosecond contrast monitor. The attenuated beam is focused in vacuum and then re-collimated using a negative and a positive lens. In this way it is possible to obtain an image of the gratings and the alignment procedure is simplified. A mirror redirects the beam to two lines, one with a relay imaging system under vacuum and the other with just a reducing beam expander. The first line is used for the autocorrelators while the second one is for other diagnostics that do not require a good quality near-field, like a streak camera or a spectrometer. A transmission through a mirror on the relayed beam is used to obtain the near-field and farfield, focusing and reimaging the beam with some lenses. The only difference for the upper table is that the first three mirrors are located in a different place, fixed by the position of the incoming beam. The different diagnostics used after the compressor are shown in Table 3.

\section{Control and performance of the diagnostics}

Each diagnostics package has a local control PC that enables the particular camera or spectrometer settings to be set. In addition, this local PC controls the setting of the motorized filter which is used to ensure the correct attenuation. These local PCs are connected to the main Vulcan laser control software via the diagnostics server software which relays commands from the control software. The control software communicates the shot type to the diagnostics server which then broadcasts this to the local diagnostics PCs; these then make the required adjustments to the settings depending on the level of attenuation required. After the laser shot the captured data are stored on the local PC and also archived on a networked drive so that they can be retrieved throughout the laser facility. After the shot the images from the diagnostics are automatically processed dependent on the diagnostic. For example, the images from the autocorrelators are analysed to give a value for the pulse duration. Figure 19 shows a histogram of the pulse durations recorded during a 53 shot experimental campaign in TAP; for all of the shots 


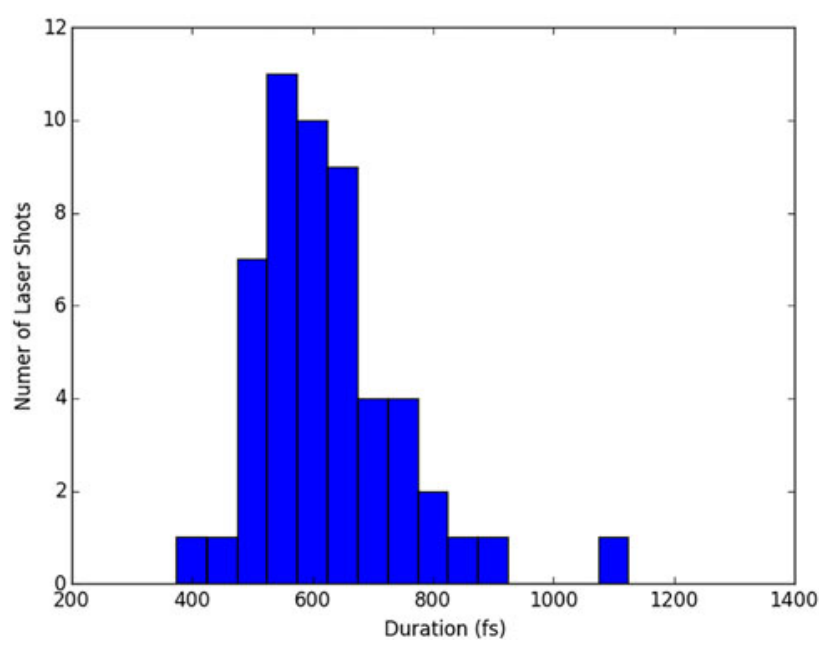

Figure 19. Histogram of pulse durations recorded during an experimental campaign in TAP.

an autocorrelation was recorded and analysed. As can be seen, the measured value varies on a shot by shot basis, with the majority of shots being between 500 and $650 \mathrm{fs}$. The equivalent histogram for an 81 shot experimental campaign for TAW is shown in Figure 20. For this dataset there is an obvious peak at $1.45 \mathrm{ps}$ with a long tail extending beyond 2 ps. The data points in the $0-100$ ps bin are due to the autocorrelator capturing a camera image, but without there being energy fired on that beam. The absence of energy was due to an intermittent trigger error during that experimental campaign.

\section{Conclusions}

In conclusion, we have presented a review of the laser diagnostic provision on the Vulcan laser. We have demonstrated a number of strategies that we have employed to enable the diagnosis of laser pulses that are generated in a wide parameter space. These strategies show how to handle the laser pulse energy in a way that minimizes the accumulation of nonlinear effects and thereby maintain the fidelity of the pulse being measured. We have demonstrated the use of a sub-aperture beam on a large aperture laser system to reliably measure the pulse duration of the laser. In addition, we have shown how we use diagnostics packages throughout the laser chain to evaluate the performance of the different subassemblies within the laser.

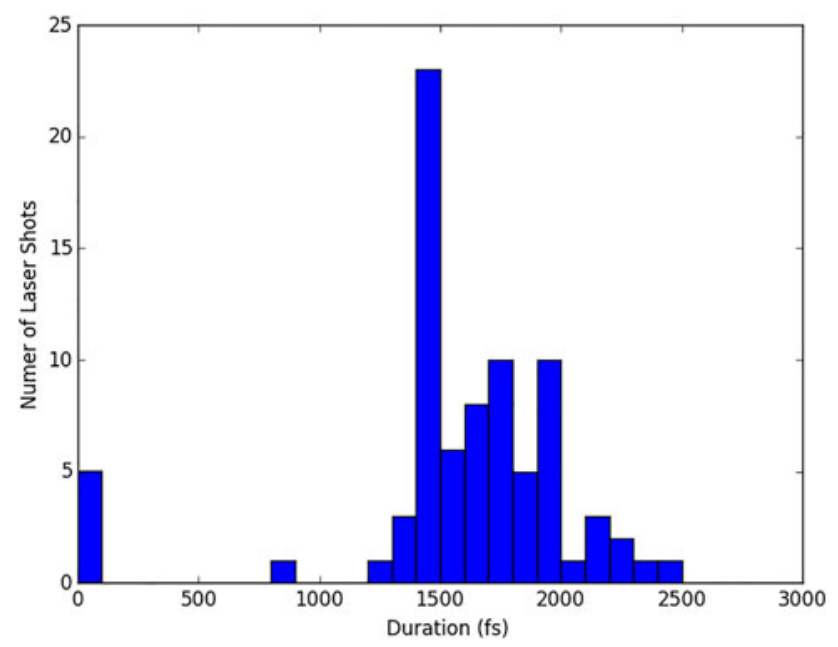

Figure 20. Histogram of pulse durations recorded during an experimental campaign in TAW.

\section{References}

1. C. Hernandez-Gomez, P. A. Brummitt, D. J. Canny, R. J. Clarke, J. Collier, C. N. Danson, A. M. Dunne, B. Fell, A. J. Frackiewicz, S. Hancock, S. Hawkes, R. Heathcote, P. Holligan, M. H. R. Hutchinson, A. Kidd, W. J. Lester, I. O. Musgrave, D. Neely, D. R. Neville, P. A. Norreys, D. A. Pepler, C. J. Reason, W. Shaikh, T. B. Winstone, and B. E. Wyborn, J. Phys. IV 133, 555 (2006).

2. C. N. Danson, P. A. Brummitt, R. J. Clarke, J. L. Collier, B. Fell, A. J. Frackiewicz, S. Hawkes, C. Hernandez-Gomez, P. Holligan, M. H. R. Hutchinson, A. Kidd, W. J. Lester, I. O. Musgrave, D. Neely, D. R. Neville, P. A. Norreys, D. A. Pepler, C. J. Reason, W. Shaikh, T. B. Winstone, R. W. W. Wyatt, and B. E. Wyborn, Laser Particle Beams 23, 87 (2005).

3. C. N. Danson, P. A. Brummitt, R. J. Clark, B. Fell, A. J. Frackiewicz, S. Hawkes, C. Hernandez-Gomez, P. Holligan, M. H. R. Hutchinson, A. Kidd, W. J. Lester, I. O. Musgrave, D. Neely, D. R. Neville, P. A. Norreys, D. A. Pepler, C. J. Reason, T. B. Winstone, R. W. W. Wyatt, and B. E. Wyborn, IAEA J. Nuclear Fusion 44, S239 (2004).

4. I. Musgrave, W. Shaikh, M. Galimberti, A. Boyle, C. Hernandez-Gomez, K. Lancaster, and R. Heathcote, Appl. Opt. 49, 6558 (2010).

5. C. Hernandez-Gomez, S. P. Blake, C. Burton, R. J. Clarke, J. L. Collier, B. Costello, V. Dubrosky, A. J. Frackiewicz, M. Galimberti, S. Hancock, S. J. Hawkes, R. Heathcote, A. K. Kidd, I. O. Musgrave, D. Neely, M. M. Notley, B. T. Parry, D. A. Pepler, W. Shaikh, T. B. Winstone, and B. E. Wyborn, CLF Annual Report 2007-2008, 260 (2008).

6. J. Collier, C. Danson, C. Johnson, and C. Mistry, Rev. Sci. Instrum. 70, 1599 (1999).

7. A. Boyle and M. Galimberti, Rev. Sci. Instrum. (to be submitted). 